\title{
SOLID $k$-VARIETIES AND HENSELIAN FIELDS
}

\author{
BY \\ GUSTAVE EFROYMSON
}

\begin{abstract}
Let $k$ be a field with a nontrivial absolute value. Define property $(*)$ for $k$ : Given any polynomial $f(x)$ in $k[x]$ with a simple root $a$ in $k$; then if $g(x)$ is a polynomial near enough to $f(x), g(x)$ has a simple root $\beta$ near a. A characterization of fields with property $(*)$ is given. If $Y$ is an affine $k$ variety, $Y \subset \bar{k}(n)$, define $Y_{k}=Y \cap k(n)$. Define $Y$ to be solid if $I(Y)=I\left(Y_{k}\right)$ in $k\left[x_{1}, \cdots, x_{n}\right]$. If $\pi: Y \rightarrow \vec{k}^{d}$ is a projection induced by Noether normalization, and if $k$ has property $(*)$, then $Y$ is a solid $k$-variety if and only if $\pi\left(Y_{k}\right)$ contains a sphere in $k^{d}$. Using this characterization of solid $k$-varieties and Bertini's theorem, a dimension theorem is proven.
\end{abstract}

0. Introduction. In [3], D. Dubois and the author proved a dimension theorem for real primes. The method used in that paper is here extended to take care of $p$-adic type fields. The idea is rather simple. Let $k$ be a field with a nontrivial absolute value $\mid: k \rightarrow R$ satisfying property $(*)$ (see $\$ 1$ ). Property $(*)$ essentially says that if a polynomial $f(x)$ in $k[x]$ has a simple root $\alpha$ in $k$, and if $g(x)$ is a polynomial of the same degree as $f(x)$ which is "near" $f(x)$, then $g(x)$ has a simple root $\beta$ near $a$. It is this property of real closed fields which enabled us to prove the main theorem of [3].

In $\$ 2$ we define a solid $k$-variety to be an (affine) $k$-variety that is determined by its $k$-points. If $k$ is a field with property $(*)$, we show that a $k$-variety $Y$ is solid if and only if any projection $\pi: Y \rightarrow \bar{k}^{d}$ induced by Noether normalization has the property that $\pi\left(Y_{k}\right)$ contains a "sphere" in $k^{d}$. We define $Y_{k}$ to be the $k$-points of $Y$. As in [3], one can prove a dimension theory and this is done in $\$ 3$.

In $\$ 1$ a characterization is given of fields with absolute value satisfying (*). If the absolute value is archimedian, the field is real closed or algebraically closed. If the absolute value is not archimedian, the field is Henselian.

In the final section some examples are given to show that if $k$ is the quotient field of the Henselization of a localized ring of algebraic numbers, then there exist $k$-varieties with no $k$-points.

1. Henselian fields and real closed fields. Let $k$ be a field with an absolute

Received by the editors February 5, 1971.

AMS 1970 subject classifications. Primary 14G20, 13J15; Secondary 13C15, 13 A 15.

Key words and phrases. Henselian field, absolute value, $k$-variety, real closed field, Noether normalization, dimension theorem. 
value $\mid: k \rightarrow R$ (the real numbers). We assume || is nontrivial, i.e. takes on values other than 0 and 1 .

Denote by $(*)$ the following property of $k,||$ :

(*) Given

(i) $f(x)=\sum_{i=0}^{n} a_{i} x^{i}$ in $k[x], a_{n} \neq 0$,

(ii) $\alpha$ in $k$, a simple root of $f(x)$,

(iii) $\epsilon>0$;

then there exists $\delta>0$, depending on $f, a$ and $\epsilon$, such that if $g(x)=\sum_{j=0}^{n} b_{j} x^{j}$ with $\left|b_{j}-a_{j}\right|<\delta$ for all $j$, then $g(x)$ has a simple root $\beta$ in $k$ and $|\beta-\alpha|<\epsilon$.

If the absolute value on $k$ is nonarchimedian, we let $A=\{a \in k|| a \mid \leq 1\}$ and $m=\{a \in k|| a \mid<1\}$. Then $A, m$ is the valuation ring of the absolute value. We define $\nu: k \rightarrow R$, the associated valuation, by $\nu(a)=-\log _{\boldsymbol{e}}|a|$.

Theorem 1. Let $k,||, A, m$, etc. be as above.

(a) If the absolute value is not archimedian, the following are equivalent:

(1) $A, m$ is Henselian.

(2) Given any $f(x)$ in $A[x], f(x)=x^{n}+\sum_{j=0}^{n-1} a_{j} x^{j}$ with simple root $a$ in $A$, and given $\epsilon>0$; there exists $\delta>0$ such that if $g(x)=x^{n}+\sum_{j=0}^{n-1} b_{j} x^{j}$ and $\left|b_{i}-a_{i}\right|<\delta, i=0, \cdots, n-1$, then $g(x)$ bas a simple root $\beta$ in $A$ and $|\beta-\alpha|<\epsilon$. (3) $k,||$ has property $(*)$.

(b) If the absolute value is archimedian, then $k$ bas property $(*)$ if and only if $k$ is real closed or algebraically closed.

Proof. (a) We show first that (1) implies (2). Following Lang [7, Proposition 2, p. 29], we find a root for $g(x)$ in the completion of $A$ and then apply a result of Nagata to conclude the root is in $A$.

Let $f(x)=(x-\alpha), b(x)$. Then $f^{\prime}(\alpha)=b(\alpha)$. Let $\left|f^{\prime}(a)\right|=|b(\alpha)|=d$. We can assume $\epsilon<1$ and now choose $\delta=d^{2} \epsilon$. Then if $g(x)$ is of the required form, $g(x)=$ $(x-a) b(x)+c l(x)$ where $l(x) \in A[x]$ and $|c|<d^{2} \epsilon$. Then $g^{\prime}(\alpha)=b(\alpha)+c l^{\prime}(\alpha)$ and so $\left|g^{\prime}(\alpha)\right|=d$. Also $g(\alpha)=c l(\alpha)$ so $|g(\alpha)| \leq d^{2} \epsilon$ and $\left|g(\alpha) / g^{\prime}(\alpha)\right| \leq$ $d^{2} \epsilon / d=d \epsilon$.

Now let $\beta_{0}=\alpha, \beta_{i}=\beta_{i-1}-g\left(\beta_{i-1}\right) / g^{\prime}\left(\beta_{i-1}\right)$. By induction we can show $\left|\beta_{i+1}-\beta_{i}\right| \leq d \epsilon^{2^{i}},\left|g\left(\beta_{i}\right)\right| \leq d^{2} \epsilon^{2}{ }^{i}$. When $i=0,\left|\beta_{1}-\beta_{0}\right| \leq d \epsilon,\left|g\left(\beta_{0}\right)\right| \leq d^{2} \epsilon$. Now suppose $\left|\beta_{i}-\beta_{i-1}\right| \leq d \epsilon^{2^{i-1}},\left|g\left(\beta_{i-1}\right)\right| \leq d^{2} \epsilon^{2^{i-1}}$. Then $\left|\beta_{i+1}-\beta_{i}\right|=$ $\left|g\left(\beta_{i}\right) / g^{\prime}\left(\beta_{i}\right)\right|$. But $g\left(\beta_{i}\right)=g\left(\beta_{i-1}\right)+\left(\beta_{i}-\beta_{i-1}\right) g^{\prime}\left(\beta_{i-1}\right)+\left(\beta_{i}-\beta_{i-1}\right)^{2}[\cdots]$ since $(1 / l) f^{(l)}(x)$ is in $A[x]$ for all $l$. So $g\left(\beta_{i}\right)=\left(\beta_{i}-\beta_{i-1}\right)^{2}[\ldots]$ and so $\left|g\left(\beta_{i}\right)\right| \leq$ $d^{2} \epsilon^{2^{i}}$. Similarly $\left|g^{\prime}\left(\beta_{i}\right)\right|=\left|g^{\prime}\left(\beta_{i-1}\right)\right|=\left|g^{\prime}\left(\beta_{0}\right)\right|=d$. So $\left|\beta_{i+1}-\beta_{i}\right| \leq d^{2} \epsilon^{2^{i}} / d=$ $d \epsilon^{2}$. Let $\hat{A}$ be a completion of $A$ with respect to || . Then we have $\lim _{i \rightarrow \infty} \beta_{i}$ $=\beta$ is a root of $g(x)$. But by Nagata [9, Theorem 12, p. 31], any element in $\hat{A}$ which is separably algebraic over $A$ is in the Henselization of $A$. So $\beta \in A$ since, by assumption, $A$ is Hensel. Also $|\beta-\alpha| \leq d \epsilon \leq \epsilon$. 
(3) $\Rightarrow(2)$ is trivial.

(2) $\Rightarrow(3)$. Let $f(x)=\sum_{i=0}^{n} a_{i} x^{i}, a_{i} \in k$. By multiplying by a nonzero constant, we can put all $a_{i}$ in $A$. Since this operation is bicontinuous, we can assume $f(x)$ $=\sum_{i=0}^{n} a_{i} x^{i}$, all $a_{i} \in A, a_{n} \neq 0$.

Now $\left(a_{n}\right)^{n-1} f(x)=\left(a_{n} x\right)^{n}+a_{n-1}\left(a_{n} x\right)^{n-1}+\cdots+a_{n-j} a_{n}^{j-1}\left(a_{n} x\right)^{n-j}+$ $\cdots+a_{0} a_{n}^{n-1}=b\left(a_{n} x\right)$ where $b(x)=x^{n}+\sum_{j=1}^{n} a_{n-j} a_{n}^{j-1} x^{n-j}$.

If $b_{n} x^{n}+b_{n-1} x^{n-1}+\cdots+b_{0}=g(x)$, let $l(x)=x^{n}+\sum_{j=1}^{n} b_{n-j} b_{n}^{j-1} x^{n-j}$. Suppose $f(x)$ has a root $\alpha \in k$. Then $b(x)$ has a root $a_{n} \alpha$ which must be in $A$. Then if $\epsilon^{\prime}<\epsilon\left|b_{n}\right|$, there exists $\delta^{\prime}$ so that if $\left|b_{n-j} b_{n}^{j-1}-a_{n}^{j-1} a_{n-j}\right|<\delta^{\prime}$, then $l(x)$ has a root $\beta^{\prime}$ with $\left|\beta^{\prime}-a_{n} \alpha\right|<\epsilon^{\prime}$. We can also assume $\left|b_{n}-a_{n}\right||\alpha|<\epsilon^{\prime}$. Then let $\beta^{\prime}=b_{n} \beta$ and then $\left|b_{n} \beta-a_{n} \alpha\right|<\epsilon^{\prime}$, and so $\left|b_{n} \beta-b_{n} \alpha\right|<\epsilon^{\prime}$ and $|\beta-\alpha|<\epsilon$.

Choose $\delta$ so that $\left|b_{i}-a_{i}\right|<\delta$ for all $i$ implies $\left|b_{n}-a_{n}\right||\alpha|<\epsilon^{\prime}$ and $\left|b_{n-j} b_{n}^{j-1}-a_{n}^{j-1} a_{n-j}\right|<\delta^{\prime}$, which is certainly possible.

$(2) \Rightarrow(1)$. By Nagata $[8,(43.2)]$, it is enough to show that if $f(x)=x^{n}+$ $\sum_{i=0}^{n-1} a_{i} x^{i}$ is in $A[x], a_{0}$ in $m, a_{1}$ not in $m$, then $f$ has a root $\alpha$ in $m$.

Suppose $f$ does not have such a root, then one can assume $f$ is irreducible by taking an irreducible factor of $\underset{\sim}{f}$ of degree $>1$ which has $a_{0}$ in $m, a_{1}$ not in $m$. This $f$ will have a root $\alpha$ in $\widetilde{A}$, the Henselization of $A$. Moreover $\alpha$ is a simple root since $a_{1} \notin \mathrm{m}$.

We let $L$ be a splitting field for $f(x)$ over $k$ and assume $k[\alpha] \subset L$. Let $\nu_{1}$, $\cdots, \nu_{m}$ be the extensions of the valuation $\nu$ to $L$. Let $H=G(L / k[\alpha])$ be the Galois group. Let $\sigma_{1}, \cdots, \sigma_{n}$ be elements of $G(L / k)$ such that $\sigma_{1} H, \cdots, \sigma_{n} H$ is a complete set of coset representatives of $G / H$. Let $\sigma_{1}$ be the identity. Then

$$
f(x)=\prod_{i=1}^{n}\left(x-\sigma_{i} \alpha\right) .
$$

Now $\nu_{1} \circ \sigma_{i}=\nu_{i}$ for some $i$. Let $\nu_{1}$ be chosen so $\nu_{1}(\alpha)>0$. This is possible since $a_{0} \in m$. Now if $\sigma \in G(L / k)$ and $\nu_{1} \circ \sigma=\nu_{1}$, we have $\sigma \in H$. For if $\sigma \notin H$, then $\sigma \alpha \neq \alpha$ and $\nu_{1}(\sigma \alpha)=0$ (since $a_{1} \notin m$ ). So let $\nu_{1}, \cdots, \nu_{s}$ be the different valuations in the set $\left\{\nu_{1} \circ \sigma_{i}\right\}$ of valuations restricted to $k[\alpha]$ and suppose $\nu_{i}$ appears $t_{i}$ times.

Now choose $\gamma_{1}=0 ; \gamma_{2}, \cdots, \gamma_{s}$ all units in $A$. Let $\epsilon=1$ and $g(x)=$ $\Pi_{i=1}^{s}\left(x-\gamma_{i}\right)^{t_{i}}$. Note that $t_{1}=1$ by the argument given above. Let $\delta$ be the delta assigned to $g(x), \gamma_{1}, \epsilon$ by (2). Let ||$_{i}$ be the absolute value associated to $\nu_{i}$. By the approximation theorem [2, p. 136], we can find $\beta$ in $k[\alpha]$ such that $\left|\beta-\gamma_{i}\right|_{i}<\delta, i=1, \cdots, s$. Let $\Pi_{i=1}^{n}\left(x-\sigma_{i} \beta\right)=b(x)$. Then $b(x) \in A[x]$ since $\beta \in A[\alpha]$, and $\left|\sigma_{i}(\beta)-\gamma_{i}\right|_{1}=\left|\beta-\gamma_{i}\right|_{i}<\delta, i=1, \cdots, n$. Thus $b(x)$ is near enough to $g(x)$ to have a root $\gamma$ in $A$ and $|\gamma|<\epsilon$ which means $\gamma$ is in $m$ and so $\gamma=\beta$ since, by construction, all other roots of $b$ are units. But $\sigma_{i} \beta \neq \beta$ for $i>1$ and so $\beta$ cannot be in $A$, a contradiction. 
(b) In [3] it is shown that a real closed field has property $(*)$. A sketch of the proof follows. Let $f(x)$ have a simple root $\alpha$ in $k$. Then $f^{\prime}(\alpha) \neq 0$ and we can find $\gamma \in k$ so that $|\gamma|<\epsilon$ and $f(\alpha-\gamma)<0<f(\alpha+\gamma)$. By choosing $\delta$ small enough, we can be sure that if $g(x)$ is chosen as in $(*)$, we have $g(\alpha-\gamma)$ $<0<g(\alpha+\gamma)$ and so $g(x)$ has a root $\beta$ with $|\beta-\alpha|<\epsilon$.

It is obvious that an algebraically closed field has proper ty $(*)$.

Now assume $k$ has property $(*)$. By Ostrowski's theorem [2, p. 131], it is known that the given absolute value is equivalent to one induced by an embedding $\sigma$ of $k$ in $R$ or $C$ following by the usual absolute value on $R$ or $C$.

Case 1 . We suppose $\sigma$ maps $k$ into $R$ and so we can consider $k$ as a subfield of $R$. If $\alpha$ is a real number algebraic over $k$, we need only show $\alpha \in k$. Let $f(x)=\operatorname{Irr}(\alpha, k, x)=\Pi_{i=1}^{n}\left(x-\alpha_{i}\right)$ in $C[x]$. We can regard $\sigma_{1}$ as the embedding $\sigma_{1}: k[\alpha] \hookrightarrow C, \sigma_{1}(\alpha)=\alpha_{1}$. The other embeddings of $k[\alpha] \hookrightarrow C$ are given by $\sigma_{i}(\alpha)$ $=a_{i}$. Now let ||$_{i}$ equal ||$\circ \sigma_{i}: k \rightarrow R\left(\left|a+b_{i}\right|=\left(a^{2}+b^{2}\right)^{1 / 2}\right)$. We easily see ||$_{1}$ is not equivalent to any of the other ||$_{i}$. Let ||$_{1},||_{2}, \cdots,||_{s}$ be the inequivalent absolute values and let $t_{i}=1$ if $\sigma_{i}(k) \subset R$, and $t_{i}=2$ if not.

Choose $\gamma_{1}, \cdots, \gamma_{s}$ in $k$ so $\gamma_{1} \neq \gamma_{i}$ if $i>1$. Choose $\epsilon<\min \left(\left|\gamma_{1}-\gamma_{i}\right| / 2\right)$ and let $g(x)=\Pi_{i=1}^{s}\left(x-\gamma_{i}\right)^{t_{i}}$. Let $\delta$ be the delta assigned by $(*)$ to $g(x), \gamma_{1}, \epsilon$, and assume $\delta<\epsilon$. By the approximation theorem [2, p. 136], given $\delta^{\prime}>0$ we can find $\beta \in k[\alpha]$ so that $\left|\beta-\gamma_{i}\right|_{i}<\delta^{\prime}=\delta / n$ for $i=1, \cdots, s$. Then $\left|\sigma_{i}(\beta)-\gamma_{i}\right|<\delta^{\prime}$.

Let $b(x)=\Pi_{i=1}^{n}\left(x-\sigma_{i}(\beta)\right)$. Then we can choose $\delta^{\prime}$ so that $b(x)$ is within $\delta$ of $g(x)$. Then $h(x)$ has a root $\gamma$ in $k$ within $\epsilon$ of $\gamma_{1}$, but this implies $\gamma=\beta$. We now get the contradiction; $\beta$ is in $k$ and $\sigma_{2} \beta \neq \beta$.

Before we consider the next case we prove

Lemma. Let $\sigma: k \hookrightarrow C$ be an embedding inducing the absolute value || on $k$. Let $\tau: C \rightarrow C$ be any automorphism and let $|a|_{\tau}=|\tau(a)|$. Then $k,||$ bas property $(*)$ if and only if $k,||_{\tau}$ does.

Proof. Assume $k,||$ has property $(*)$. Identify $k$ with $\sigma(k)$. Let $f(x)=$ $\sum_{i=0}^{n} a_{i} x^{i}$ be in $k[x]$ with simple root $\alpha$ in $k$. Then $f^{\tau}(x)=\sum_{i=0}^{n} \tau\left(a_{i}\right) x^{i}$ has simple root $\tau(\alpha)$. By assumption, there exists $\delta$ such that if $g(x)=\sum_{i=0}^{n} b_{i} x^{i}$ where $\left|b_{i}-a_{i}\right|_{\tau}<\delta$, then $g^{\tau}(x)$ has a simple root $\beta^{\prime}$, where $\left|\beta^{\prime}-\tau(\alpha)\right|<\epsilon$ (since $\left|b_{i}-a_{i}\right|_{\tau}<\delta$ if and only if $\left|\tau\left(b_{i}\right)-\tau\left(a_{i}\right)\right|<\delta$ ). Let $\beta=\tau^{-1}\left(\beta^{\prime}\right)$. Then $|\tau(\beta)-\tau(\alpha)|<\epsilon$ and so $|\beta-\alpha|_{\tau}<\epsilon$ and $\beta$ is a simple root of $g(x)$.

Now back to $k \hookrightarrow G$. We wish to show we can assume Case 2: There is a subfield $b$ of $k$ so that $k$ is algebraic over $b$ and $\sigma(b) \subset R$. To do this, let $\left\{t_{i}\right\}_{i \in I}$ be a transcendence basis for $k$ over $Q$. Choose $\left\{u_{i}\right\}_{i \in I}$ as an algebraically independent set in $R$ and define $\tau: Q\left(\left\{t_{i}\right\}\right) \hookrightarrow R$ so $\tau\left(t_{i}\right)=u_{i}$. Extend $\tau$ to $r: k \hookrightarrow C$ and let $b=Q\left(\left\{t_{i}\right\}\right)$. If the $u_{i}$ are chosen correctly, there exists an automorphism $\mu$ of $C$ so that $\tau=\mu \circ \sigma: k \hookrightarrow C$. Replace || by ||$_{\mu}$ and we have 
Case 2. There is a subfield $b$ of $k$ such that $\sigma(b) \subset R$ and $k$ is algebraic over $b$. We can consider then $b \subset k \subset C, b \subset R$. Take $a \in R, \alpha$ algebraic over $k$. Then $\operatorname{Irr}(\alpha, k, x)$ has a real root $\alpha$. We can now apply the same argument as in Case 1 to conclude $a \in k$. Thus $k$ contains a real closure of $b$ in $C$ and so either $k$ is real closed or algebraically closed. Q.E.D.

2. Solid $k$-varieties. Let $k$ be a field, $\bar{k}$ its algebraic closure. A $k$-algebraic set is a subset of $\bar{k}^{n}$ which is the set of zeros of some ideal $I \subset\left[X_{1}, \cdots, X_{n}\right]$. A variety $Y$ is an absolutely irreducible algebraic set (i.e., the algebraic set determined by $I(Y) \otimes \bar{k}$ in $\bar{k}\left[X_{1}, \cdots, X_{n}\right]$ is still $Y$ and cannot be written as the union of two smaller algebraic sets in $\bar{k}^{n}$ ). In characteristic $p, Y$ must also be separable, i.e. $k(Y)$ separable over $k$. $\left.Y_{k}\right\}$.

Now let $Y_{k}=Y \cap k^{(n)}$, and $I\left(Y_{k}\right)=\left\{f \in k\left[X_{1}, \cdots, X_{n}\right] \mid f(P)=0\right.$ for all $P$ in

Definition. Let $Y$ be a $k$-variety. Then $Y$ is a solid $k$-variety if $I\left(V_{k}\right)=I(V)$.

Now let $V$ be $d$-dimensional. Let $\Gamma[Y]$ be the coordinate ring $k\left[X_{1}, \cdots, X_{n}\right] / I(Y)$. Then by Noether normalization $\left[10\right.$, p. 266], there exist $x_{1}, \cdots, x_{d}$ independent transcendentals in $\Gamma[Y]$ so that $\Gamma[Y]=k\left[x_{1}, \cdots, x_{n}\right]$ is integral and separable over $k\left[x_{1}, \cdots, x_{d}\right]$. Let $\pi: Y \rightarrow A^{d}(\bar{k})=\bar{k}^{d}$ be the associated projection. Then $\pi$ induces $\pi_{k}: V_{k} \rightarrow k^{d}$.

Now let $k$ be a field with absolute value $\mid$. We can use this absolute value to define a metric on $k^{d}$ by defining $|P-Q|=\left(\sum_{i=1}^{d}\left|x_{i}(P)-x_{i}(Q)\right|^{2}\right)^{1 / 2}$ for $P, Q$ in $k^{d}$. We define a sphere $S_{P, \epsilon}, P \in k^{d}, \epsilon>0$, to be $\left\{Q \in k^{d}|| P-Q \mid<\epsilon\right\}$.

Theorem 2. Let $k,||$ satisfy $(*)$. Let $Y$ be a k-variety of dimension $d$, $\pi: Y \rightarrow \bar{k}^{d}$ as above. Then $Y$ is a solid $k$-variety if and only if $\pi_{k}\left(Y_{k}\right)$ contains some sphere $S_{P, \epsilon}$.

Proof. First assume $Y$ is a solid $k$-variety. Let $B=k\left[x_{1}, \cdots, x_{n}\right]$. Choose $z \in B$ so that $k\left[x_{1}, \cdots, x_{d}, z\right]$ has the same quotient field as $B$. Let $f(z)=$ $\sum_{i=0}^{m} a_{i}\left(x_{1}, \cdots, x_{d}\right) Z^{i}$ be the primitive irreducible polynomial for $z$ over $k\left(x_{1}, \cdots, x_{d}\right)$. Then $f$ is irreducible in $k\left[x_{1}, \cdots, x_{d}, Z\right]$. Now $x_{d+i}=$ $\sum_{j=0}^{m-1}\left[b_{i j}\left(x_{1}, \cdots, x_{d}\right) / c_{i j}\left(x_{1}, \cdots, x_{d}\right)\right] z^{j}$ where $b_{i j}, c_{i j} \in k\left[x_{1}, \cdots, x_{d}\right]$. Let $U=$ $\left\{P^{\prime} \in Y \mid a_{m}\left(P^{\prime}\right) \neq 0\right.$, all $c_{i j}\left(P^{\prime}\right) \neq 0$, and $\left.(\partial f / \partial z)\left(P^{\prime}\right) \neq 0\right\}$. Then $U$ is Zariski open and nonempty in $Y$. Since $Y$ is solid, $U \cap Y_{k}$ is nonempty. Choose $P^{\prime} \in U \cap Y_{k}$ and let $P=\pi\left(P^{\prime}\right)$. Then $z\left(P^{\prime}\right)$ is a simple root of $f_{P}(Z)=\sum_{i=0}^{m} a_{i}(P) Z^{i}$. Now choose $\epsilon$ small enough so that, if $Q \in k^{d}$ and $|Q-P|<\epsilon$, we have

(1) all $c_{i j}(Q) \neq 0$,

(2) $a_{m}(Q) \neq 0$

(3) $f_{Q}(Z)=\sum_{i=0}^{m} a_{i}(Q) Z^{i}$ has a simple root $a \in k$.

To get (3) we use the $(*)$ condition. 
We will now show if we define

$$
Q^{\prime}=\left(x_{1}(Q), \cdots, x_{d}(Q), x_{d+1}(Q, \alpha), \cdots, x_{n}(Q, \alpha)\right)
$$

that $Q^{\prime}$ is a point of $Y_{k}$ with $\pi\left(Q^{\prime}\right)=Q$.

To see this, note $/$ irreducible in $k\left[x_{1}, \cdots, x_{d}, Z\right]$ implies $(f)$ is prime in this ring. But $Z \rightarrow z$ induces a homomorphism $k\left[x_{1}, \cdots, x_{d}, Z\right] \rightarrow k\left[x_{1}, \cdots, x_{d}, z\right]$ which is onto. Since the image is an integral domain, the kernel must be a prime ideal containing $(f)$, but by looking at dimensions, we see the kernel must be $(f)$. So $x_{i} \rightarrow x_{i}(Q), Z \rightarrow \alpha$, induces a homomorphism $k\left[x_{1}, \cdots, x_{d}, z\right] \rightarrow k$. This homomorphism extends to a homomorphism $B \rightarrow k$ since all $c_{i j}(Q) \neq 0$ and this corresponds to a point of $Y_{k}$ which is just $Q^{\prime}$. This shows $\pi\left(Y_{k}\right) \supset S_{P, \epsilon}$.

Now assume $\pi_{k}\left(Y_{k}\right) \supset S_{P, \epsilon^{\bullet}}$

We prove the following lemmas.

Lemma 1. If $f\left(x_{1}, \cdots, x_{d}\right) \in k\left[x_{1}, \cdots, x_{d}\right]$ and $f\left(S_{P, \epsilon}\right)=0$ for some sphere, then $f=0$.

Proof. By induction on $d$. If $d=1, f(x)$ has an infinite number of roots and so $f=0$.

For $d>1$, we can assume $P=(0, \cdots, 0)$ without loss of generality. Fix $a$ with $|a|<\epsilon$. For $g\left(x_{1}, \cdots, x_{d-1}\right)=f\left(x_{1}, \cdots, x_{d-1}, a\right)$, we have $g(Q)=0$ if $|Q|$ $<\epsilon-|a|$, and so by.induction $g \equiv 0$. But then for each fixed $a_{1}, \cdots, a_{d-1}$, $f\left(a_{1}, \cdots, a_{d-1}, x\right)$ has an infinite number of roots and is identically zero.

Lemma 2. Let $Y$ be a variety of dimensiond and let $\sigma: V \rightarrow A^{d}(\bar{k})$ be a morphism such that $\sigma_{k}\left(Y_{k}\right) \supset S_{P, \epsilon}$. Then $Y$ is solid.

Proof. If $Y$ is not solid, then there exists an algebraic set $W$ of codimension one in $Y$ such that $W \supset V_{k}$. But then $\sigma(W) \supset \sigma\left(V_{k}\right) \supset \sigma_{k}\left(V_{k}\right) \supset S_{P, \epsilon^{\bullet}}$ But $\sigma(W)$ is of codimension at least 1 in $A^{d}(\bar{k})$ and so there exists $f \in k\left[x_{1}, \cdots, x_{d}\right]$ such that $f(\sigma(W))=0$ which contradicts Lemma 1.

Now apply Lemma 2 to $\pi$ and we see $Y$ is solid.

\section{The dimension theorem for solid $k$-varieties.}

Theorem 3. Let $k$ be a field with an absolute value satisfying (*). Let $Y$ be a solid k-variety; $W_{1}, \cdots, W_{r}$ subvarieties of $Y$ of codimension at least 2. Then there exists a solid k-variety $W$ of codimension 1 in $Y$ such that $W \supset W_{1}$ $\cup \ldots \cup W_{r}$.

Proof. Let $\pi: Y \rightarrow A^{d}(\bar{k}), d=\operatorname{dim} Y$ be as in $\$ 2$. Then $\pi$ is surjective. We let $\pi\left(W_{i}\right)=Z_{i}$. By [3, Proposition 2], there exist $g_{1}, \cdots, g_{s}$ in $k\left[x_{1}, \cdots, x_{d}\right]$ such that 
(1) $g_{i}\left(Z_{j}\right)=0$ for all $i, j$;

(2) $\operatorname{tr} \operatorname{deg}_{k} k\left(g_{1}, \cdots, g_{s}\right) \geq 2$;

(3) $g_{1}, \cdots, g_{s}$ have no common component.

Actually Proposition 2 in [3] was proved with the assumption of characteristic zero, but this restriction is easily removed.

By Theorem 2, there exists a sphere $S_{P, \epsilon} \subset \pi_{k}\left(Y_{k}\right)$. We can arrange it so that $S_{P, \epsilon} \cap\left(\bigcup_{i=1}^{r} Z_{i}\right)=\varnothing$. Now choose $f \in k\left[x_{1}, \cdots, x_{d}\right]$ so that $f(P) \neq 0$ and $f\left(Z_{j}\right)=0$, all $j$. We also choose $f$ of degree in $x_{d}$ greater than that of all the $g_{i}$ 's.

Let $f\left(x_{1}, \cdots, x_{d}\right)=\sum_{i=0}^{m} a_{i}\left(x_{1}, \cdots, x_{d-1}\right) x_{d}^{i}$. Then on a Zariski open set of $A^{d-1}(\bar{k})$, we have $a_{m}\left(x_{1}, \cdots, x_{d-1}\right) \neq 0$. We can change $S_{P, \epsilon}$ again so that $a_{m}\left(x_{1}(P), \cdots, x_{d-1}(P)\right) \neq 0$.

Consider the map $\sigma: A^{d}(\bar{k}) \rightarrow A^{d-1}(\bar{k})$ given by $\sigma:\left(x_{1}, \cdots, x_{d}\right) \rightarrow\left(x_{1}, \cdots, x_{d-1}\right)$. Then we have $a_{m}(\sigma(P)) \neq 0$.

Now multiply $f$ by $x_{d}-x_{d}(P)$ and we get a new $f$ with $f\left(Z_{i}\right)=0$, all $i$, and moreover $x_{d}(P)$ is a simple root of $f\left(x_{1}(P), \cdots, x_{d-1}(P), x_{d}\right)$.

Consider the linear system $f+\sum_{i=1}^{s} \lambda_{i} g_{i}$ which can be considered as a linear system on $Y$ or on $A^{d}(\bar{k})=\pi(Y)$. Then

$$
\begin{aligned}
f+\sum_{i=1}^{s} \lambda_{i} g_{i} & =a_{m}\left(x_{1}, \cdots, x_{d-1}\right) x_{d}^{m+1}+\sum_{i=0}^{m} c_{i}\left(x_{1}, \cdots, x_{d-1}, \lambda_{1}, \cdots, \lambda_{s}\right) x_{d}^{i} \\
& =b\left(\lambda_{1}, \cdots, \lambda_{s}, x_{1}, \cdots, x_{d}\right)
\end{aligned}
$$

and

$$
a_{m}(\sigma(P)) \neq 0 .
$$

Since the $a_{m}$ and the $c_{i}$ are continuous in $x_{1}, \cdots, x_{d}, \lambda_{1}, \cdots, \lambda_{s}$; we can find spheres $S_{\sigma(P), \epsilon^{\prime}}$ in $k^{d}$ and $S_{0, \epsilon^{\prime \prime}}$ in $k^{s}$ so that

If $\left(b_{1}, \cdots, b_{d-1}\right)$ is in $S_{\sigma(P), \epsilon^{\prime}}$ and $\left(\lambda_{1}, \cdots, \lambda_{s}\right)$ is in $S_{0, \epsilon^{\prime \prime}}$, then $b\left(\lambda_{1}, \cdots, \lambda_{s}, b_{1}, \cdots, b_{d-1}, x_{d}\right)$ as a polynomial in $x_{d}$ has a root $b_{d}$ and $\left(b_{1}, \cdots, b_{d}\right)$ is in $S_{P, \epsilon^{*}}$

Now consider $f+\sum_{i=1}^{s} \lambda_{i} g_{i}$ as a linear system on $Y$. Then by Bertini's theorem, [4] or [5] as used in [3], we see there is a Zariski neighborhood of $(0, \cdots, 0)$ in $A^{s}(\bar{k})=\bar{k}^{s}$ so that for $\left(\lambda_{1}, \cdots, \lambda_{s}\right)$ in this neighborhood, we have $V\left(f+\sum_{i=1}^{s} \lambda_{i} g_{i}\right)$ is a variety. Here $V\left(f+\sum_{i=1}^{s} \lambda_{i} g_{i}\right)$ equals the zeros of $f+$ $\sum_{i=1}^{s} \lambda_{i} g_{i}$ on $Y$. Now choose $\left(\lambda_{1}, \cdots, \lambda_{s}\right)$ in the intersection of this Zariski neighborhood with $S_{0, \epsilon}$. Then let $W=V\left(f+\sum_{i=1}^{s} \lambda_{i} g_{i}\right)$ and it is easy to check that $\sigma \circ \pi(W) \supset S_{\sigma(P), \epsilon^{\prime}}$. Then by Lemma 2,W is a solid $k$-variety and it is easy to see that it has all the properties we desire.

4. Applications when $k \subset \bar{Q}$. One can get lots of applications of Theorem 3 
by using Theorem 1 to construct fields with property $(*)$. For instance, let $K$ be a number field, that is, a finite algebraic extension of $Q$, the rationals. Then let $\mathcal{O}$ be the algebraic integer $s$ in $K$ and choose any prime $p \subset \mathcal{O}$. Then $\mathcal{O}_{p}$ is a rank 1 valuation ring. Let $\mathcal{O}_{p}$ be the Henselization of $\mathcal{O}_{p}$, and by Theorem 1 , if $k$ is the quotient field of $\tilde{\Theta}_{p}$, then $k$ has property $(*)$. Note these fields can be embedded in $\bar{Q}$, the algebraic closure of $Q$.

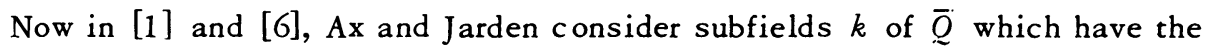
property that all $k$-varieties have points in $k$. It is obvious that this is not the case for fields constructed from number fields as the following standard examples show.

Proposition 1. Let $\mathcal{O}$ be the ring of integers in some number field, $p$ a prime in $\mathcal{O}$ and $\mathcal{O}_{p}$ the localization. Let $A$ be the Henselization of $\mathcal{O}_{p}$ and $m$ the maximal ideal of $A$. Let $k$ be the quotient field of $A$. Then there exists a projective (and bence also an affine) $k$-curve which bas no points in $k$.

Proof. Note $A / m A \cong \mathcal{O}_{p} / p \mathcal{O}_{p}$ and so is finite. We first assume that the characteristic of $A / m A$ is not 2 and that $A / m A$ is not a field with three elements. Then we can choose $\alpha \in A$ so that $\alpha \equiv 0,1$ or $2 \bmod m$. Let $q=\operatorname{order}$ of $A / m A$. Then $X^{q-1} \bmod m$ takes on only the values 0 and 1 . So the equation $X^{q-1}+$ $Y^{q-1}=\alpha Z^{q-1}$ defines a projective $k$-curve $C$ with no $k$-points. For we can multiply through so if $(X, Y, Z) \in C$, then all of $X, Y, Z$ are in $A$ and at least one is a unit. Then $X^{q-1}+Y^{q-1} \equiv 0,1$ or $2 \bmod m$ and is $\equiv 0$ if and only if $X$ and $Y$ are both not units. But $\alpha Z^{q-1} \equiv \alpha \bmod m$ if $Z$ is a unit and 0 if $Z$ is not a unit. Thus we get the contradiction that none of $X, Y, Z$ can be units.

If $A / m A$ is of characteristic 2, choose $r \geq 2$ large enough so that $m^{r} \subset(2)$. Now choose $n$ so that if $X \in A, X^{n} \equiv 0$ or $1 \bmod m^{r}$. Then choose a unit $a$ in $A$ with $\alpha \neq 1 \bmod m^{r}$. Then $X^{n}+Y^{n}=\alpha Z^{n}$ defines a projective $k$-curve with no $k$-points. Characteristic 3 is taken care of similarly.

\section{BIBLIOGRAPHY}

1. J. Ax, Solving diophantine problems modulo every prime, Ann. of Math. (2) 85 (1967), 161-183. MR 35\#126.

2. N. Bourbaki, Algèbre commutative. Chap. VI: Valuations, Actualités Sci. Indust., no. 1308, Hermann, Paris, 1964. MR $33 \# 2660$ 。

3. D. Dubois and G. Efroymson, $A$ dimension theorem for real primes (submitted).

4. A. Grothendieck with the collaboration of J. Dieudonné, Eléments de géométrie algébrique, Inst. Hautes Études Sci., Bures sur Yvette, Chapter 5 (to appear).

5. A. Grothendieck, Local properties of morphisms, A course given at Harvard University, Cambridge, Mass., 1963.

6. M. Jarden, Rational points on algebraic varieties over large number fields, Bull. Amer. Math. Soc. 75 (1969), 603-606. MR 39 \#1456. \#3974.

7. S. Lang, Algebraic numbers, Addison-Wesley, Reading, Mass., 1964. MR 28 
8. M. Nagata, Local rings, Interscience Tracts in Pure and Appl. Math., no. 13, Interscience, New York, 1962. MR 27 \#5790.

9. - On the theory of Henselian rings, Nagoya Math. J. 5 (1953), 45-57. MR 14, 529 .

10. O. Zariski and P. Samuel, Commutative algebra. Vol. I, University Series in Higher Math., Van Nostrand, Princeton, N. J., 1958. MR 19, 833.

DEPARTMENT OF MATHEMATICS AND STATISTICS, UNIVERSITY OF NEW MEXICO, ALBUQUERQUE, NEW MEXICO 87106 